\title{
A Pilot-Wave Gravity and the Titius-Bode Law
}

\author{
J.R. Croca ${ }^{1,2}$, P. Castro 2 , M. Gatta ${ }^{2,3}$, A. Cardoso ${ }^{2}$ and R. Moreira ${ }^{2}$ \\ ${ }^{1}$ University of Lisbon, Faculty of Sciences, Department of Physics \\ ${ }^{2}$ Center of Philosophy of Sciences of the University of Lisbon \\ ${ }^{3}$ CINAV and Escola Naval (Portuguese Naval Academy) \\ Email: jncroca@fc.ul.pt; jpcastro@fc.ul.pt; mariogatta@gmail.com; amb.cardoso@gmail.com; \\ ranmoreira@gmail.com
}

\begin{abstract}
Since its initial proposal in 1766, Titius-Bode empirical law has remained a puzzling source of discomfort as it predicts the average distances from the planets to the Sun for no apparent reason. Using a framework analogous to de Broglie's pilot wave theory and the self-organizing Principle of Eurhythmy, we claim that several main physical quantities describing the Solar System are quantified. Hence the Titius-Bode Law is a direct manifestation of gravitational pilot-waves at work in the Solar System.
\end{abstract}

Keywords: Titius-Bode Law, Gravity, Pilot wave theory, planet-star interactions.

\section{Titius-Bode Law History and Formulation}

The occurrence of simple numerical relations between observable quantities in natural phenomena has been a constant source of interest and even fascination, as has happened with the Pythagoreans. In some occasions such relations go beyond the purely mystical and lead to important scientific insights. Among many possible examples, one may recall de Broglie's fundamental intuition that the occurrence of integer numbers in Bohr's model, for the hydrogen atom, should have something to do with wave phenomena, for which that occurrence was well established and easily understood. This was undoubtedly one of the major impulses leading to the birth of present-day quantum mechanics.

Less successful has been the hypothesis put forward in 1766 by Johann Daniel Titius von Wittenberg for the Solar System in his German translation of the French book "Contemplation de la nature" by Charles Bonnet, and first published in 1764, in Amsterdam. In a paragraph added by himself, Titius proposes that the distances $r_{N}$ of the planets to the Sun are described approximately by a simple algebraic relation that reads,

$$
r_{N}=4+3 \times 2^{N}
$$

taking the integer $\mathrm{N}$ to be $-\infty$ for Mercury, 0 for Venus, 1 for the Earth and so on, and where at this scale the size of Earth's orbit is 10 .

In 1772, the renowned German astronomer Johann Elert Bode paid further attention to this curious relation by including a footnote in his second edition of "Anleitung zur Kentniss gestirnten des Himmels", praising the same sequence of planetary distances he had read in Titius book, although not crediting the original author for the remark. History nevertheless was generous enough to call this empirical relation the "Titius-Bode Law".

At the time of the first publication of Titius-Bode Law only six planets, up to Saturn, were known. The resulting distances would fit quite well, provided one skipped the case $\mathrm{N}=3$ where the dwarf planet Ceres was eventually discovered in 1801. Uranus also fit well the expected distance, but there were serious disagreements for Neptune and Pluto.

Various attempts to improve the applicability of the Titius-Bode Law were made over the years. The most important of these are due to the astronomers Mary Adela Blagg in 1913 [1] and E. Richardson, in $1945[2]$.

A salient fact of Blagg's formula is that the base is no longer the number 2 but 1.7275:

$$
r_{N}=A(1.7275)^{N}[B+f(\alpha+N \beta)]
$$


without an additive constant as in the previous expression. In the above, $\mathrm{A}, \mathrm{B}, \alpha$ and $\beta$ are all constants with values depending on the particular orbital system one is considering. The function $\mathrm{f}$ is periodic, with values between 0 and 1 , describing the deviations from the straight line one would obtain in a semi-log graph of distances vs. the integers $\mathrm{N}$.

Richardson's formulation, although somewhat different, shares essentially the same base for the geometric progression:

$$
r_{N}=A(1.728)^{N} \rho_{N}\left(\theta_{N}\right)
$$

where $\rho_{N}$ is a periodic function and $\theta_{N}=N(4 \pi / 13)$.

In general, both formulations provided much better agreements than any of the previous attempts, both for the solar system and for the satellites of the known planets, taking each planet as the central body.

A concordant fitting of a numerical function to the observed orbital parameters calls for the search of a possible underlying physical explanation. Following Nieto [3] one can divide such explanations for the Titius-Bode relation in three main types, viz., electromagnetic theories, gravitational theories and nebular theories.

The basic formation mechanisms of the solar system considered are either the capture by the protoSun's gravitational force of various forms of approaching material, with an agglomeration of these materials in present planets and satellites, or the condensation of a rotating, primordial gas nebula originating both the Sun and the planets. Favoring this last approach, Nieto divides the formation of the Solar system into three periods: the disk period, from which the geometric progression in Blagg's and Richardson's Laws results, the aggregation period and the planetary period, the last two corresponding to the oscillatory component in the formulas and resulting from gravitational interactions during the planetary orbital periods' stabilization.

In the beginning of the twentieth century a new set of empirical laws, by Balmer, Pashen and others, were discovered to predict the spectroscopic lines of the hydrogen atom. From these expressions it was possible to derive empirical laws describing the average distance of the electron to the nucleus of the atom. It is remarkable how both type of empirical laws, atomic and astronomical, seem to predict the distances from orbiting entities to a central body using integer numbers. At the start of quantum physics this was taken as clear evidence that a wavelike phenomenon was taking place, determining behavior of particles. Perhaps this is also the case for the Solar System.

As it happens, empirical laws for atomic physics lead to the discovery of quantum waves by de Broglie [4]. The French physicist was able to show that the appearance of a sequence of integer numbers was related to wave resonance phenomena similar to what happens with radiation in a cavity. In order for electronic orbits to be stable, their lengths must be multiples of the quantum wave half-lengths. In the same sense one can argue that the Titius-Bode integer sequences, providing the average distance of planets to the Sun, suggests the presence of stationary gravitational waves.

\section{Generalizing Titius-Bode Law}

To study the wave stability hypothesis for the Solar System we will apply Louis de Broglie's pilot wave theory, adapting it to the astronomical scale. According to de Broglie, quantum waves and corpuscles do exist simultaneously. The corpuscle vibrates on the subquantum media generating the wave, a subquantum wave, which in turn guides the corpuscle through a nonlinear process according to the now famous wavelength-linear momentum relation.

De Broglie called such perturbations on the subquantum media "pilot waves" since they would guide particles in their trajectories. De Broglie's initial model was improved upon [5] by the introduction of finite waves corresponding to what can also be named empty waves, theta waves or even subquantum waves.

Here we will try to formulate an initial gravitational wave theory also proposed in reference [5], without Newtonian forces or space-time curvature effects, consistent with the Titius-Bode relation.

In what follows, we will assume that the Sun and each one of its orbiting planets behaves like a macroscopic corpuscle, each body generating its own pilot wave or theta wave as it will be named from here. Each planetary wave is approximately confined to the planet's orbit. As with the quantum case, 
the orbital stability condition for the planet will be that its orbital perimeter equals an integer number of half-wavelengths.

Another hypothesis that we will apply is the Principle of Eurhythmy [5] a natural generalization of de Broglie guiding principle. The Principle of Eurhythmy states that in Nature each complex system adopts an average behavior that will promote the system's structural stability, allowing it to endure in time. Since no system is isolated, this means that in order to endure, each system must exhibit patterns having something in common with the patterns exhibited by nearby systems. We thus must look carefully for common features in the parameters describing the interacting systems, allowing them to synchronize their individual patterns in an overall global pattern. This global synchronization will improve the stability of all systems according to the Principle of Eurhythmy.

A key feature of this approach, relating all systems' theta waves, composing a global theta wave, is that according to the Principle of Eurhythmy, all systems must, on average, end up in places where the global theta wave has the largest intensity. Our main goal is to explain the Titius-Bode distribution as a consequence of the existence of theta waves and the Principle of Eurhythmy. Secondly, we must test the consistency of the overall wave model for the Solar System.

We start by asking why each planet orbits the Sun at a certain particular distance instead of any other. Using the idea that the behavior parameters of the Sun must have something in common with the planets' behavior parameters, a possible answer, under the Principle of Eurhythmy, is that the orbit of each planet must fulfil three conditions:

i) The planet's orbit must be located at a distance from the Sun where the intensity of the Sun's theta wave has a maximum.

ii) The planet's orbit must be located at a distance from the Sun for which the orbital perimeter equals an integer number of half-wavelengths of the planet's theta wave.

iii) As we expect the Sun theta wave to extend to its immediate vicinity, reaching the orbit of Mercury, we will assume that the wavelengths of the theta waves for the Sun and Mercury will have about the same value. More generally, we are assuming that one or more parameters describing each planet behavior must equal the same parameter describing the Sun's behavior.

The first statement is the fundamental physical instantiation of the Principle of Eurhythmy. The second statement is the condition for orbital stationarity and it may be interpreted as yet another instantiation of the Principle of Eurhythmy. The planet will be moving around the Sun with same linear velocity as its stationary wave. The third statement may be interpreted as a synchronization condition making it possible for the Sun and Mercury to hold together in an eurhythmic way, giving rise to the gravitational interaction between the two bodies. It may also be interpreted as a resonance or synchronization condition between Mercury and the Sun.

The overall stability of the Solar System will result from the conjunction of the three conditions above, defining an eurhythmic physical situation. That is, one that allows for each system, the Sun and the planets, to maintain their own structural integrity, while giving rise to a much larger and global system.

Before writing down the mathematical formulation of conditions i), ii) and iii) and working its consequences, we will gather the adequate empirical data about the Solar System. As it happens, we can use for each physical quantity of interest a quantification condition of the form

$$
Q_{N}=A e^{B N}
$$

where Q is a Solar System's physical quantity, $\mathrm{N}$ is an integer variable starting in 1, and A and B are constants derived from an exponential fitting over the set of astronomical data for Q.

In what follows we will use empirical data from NASA website [6] concerning only the first eight celestial bodies, leaving Pluto out since its empirical data does not fit (4) as well as the remaining planets. It will be assumed that additional unknown wave phenomena is taking place, deviating Pluto's behavior from the exponential distribution.

We have found that, along with the radial distance, some quantities are fairly well approached by exponential distributions in the Solar System, namely the orbital period and, consequently, also the orbital linear and angular velocities. Planetary masses and hence planetary linear and angular momenta are not quantified. 
The following graphs and expressions were obtained from an exponential fitting over a set of eight values for each quantity of interest. We started by fitting NASA's original data. After obtaining the A type constant in (4) we multiplied it by the proper values to obtain the equivalent expression in SI units.

We start by the planets' average distances from the Sun, assuming circular orbits and giving yet another expression for the Titius-Bode Law. According to NASA we have

Table 1. Planet's average distances from the Sun $\left(10^{6} \mathrm{~km}\right)$.

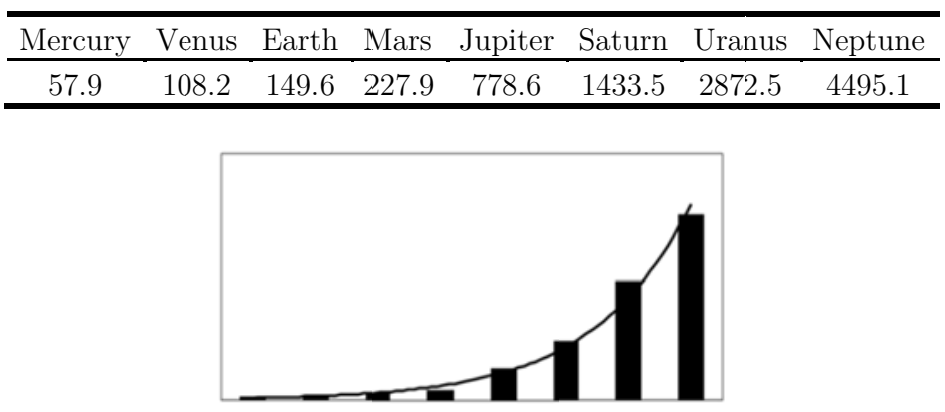

Figure 1. Exponential fitting for the planetary distances to the Sun.

The fitting expression in meters reads

$$
r_{N} \cong 25.675 \times 10^{9} e^{0.6532 N}
$$

For the orbital linear velocities in $\mathrm{km} / \mathrm{s}$, NASA gives

Table 2. Planet's orbital linear velocities $(\mathrm{km} / \mathrm{s})$.

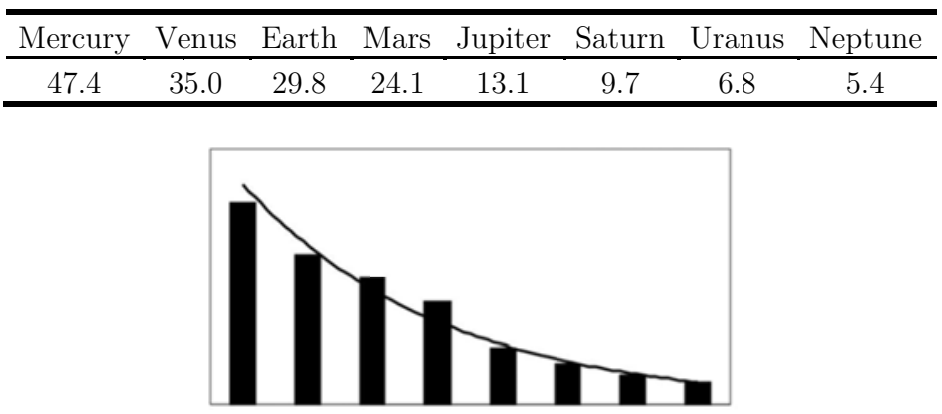

Figure 2. Exponential fitting for the planetary orbital velocities.

The fitting expression in $\mathrm{m} / \mathrm{s}$ is

$$
v_{N} \cong 71617 e^{-0.3259 N}
$$

We now consider the adequate mathematical formulation for conditions i), ii) and iii). Planetary distances follow expression (5), and therefore, according to condition i) the Sun's theta wave's intensity must have its extremes, that is, crests and valleys, distributed exponentially. This can be accomplished by letting the Sun's theta wavelength vary as follows,

$$
\theta \cong A e^{-\frac{r^{2}}{2 \sigma^{2}}} \cos \left(\frac{2 \pi r}{\alpha e^{0.6532 r}}\right)
$$

The constants A, $\sigma$ and $\alpha$ are not relevant for the line of reasoning that we will follow. Only the graphical shape of this function is of importance to our conclusions.

The first conclusion being that such a wavelet immediately explains the Titius-Bode Law. According to condition i), the planets are located at those distances from the Sun where itts theta wave has its 
crests and valleys. Since these occur along an exponential distribution from (7), we get the Titius-Bode Law.

We must now test the consistency of the overall wave model for the Solar System, and namely the consistency of condition ii) for each planet. Figure 3 below represents the Sun's theta wave intensity given by an expression of the form (7), for specifically chosen parameters,

$$
\mathrm{I}=\left(e^{-0.008 r^{2}} \cos \left(\frac{2 \pi r}{1.35 e^{0,6532 r}}\right)\right)^{2}
$$

spreading over the Solar System's orbital plane.

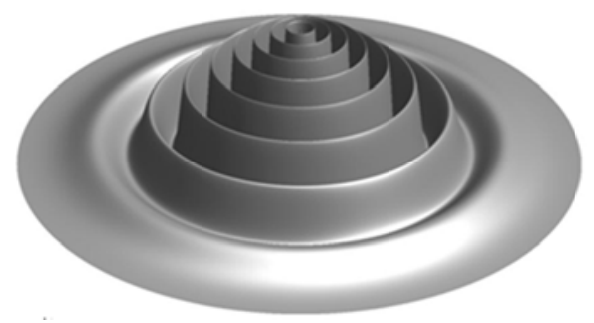

Figure 3. Sun's theta wave intensity.

Mercury is to be considered at the lowest possible orbit, located at the Sun's theta wave's first crest, that is, the first half-wavelength past the position occupied by the Sun at the center of the figure.

For Mercury's orbit, condition i) will then lead to

$$
\frac{\lambda_{S}}{2}=r_{1}
$$

where $\lambda_{S}$ is the first complete wavelength value (one crest and one valley) of the Sun's theta wave and $r_{1}$ is the distance from Mercury to the center of the Sun.

Condition ii) for Mercury's orbit allows us to write

$$
2 \pi r_{1}=n_{1} \frac{\lambda_{1}}{2}
$$

where $r_{1}$ is, as before, the distance from Mercury to the center of the Sun, $\lambda_{1}$ is Mercury's theta wave wavelength and $n_{1}$ is an integer, valid for the perimeter of Mercury's orbit.

Finally, condition iii) reads

$$
\lambda_{S}=\lambda_{1}
$$

which must be taken as an assumption.

From the three expressions above, we will get

$$
n_{1}=2 \pi \cong 6
$$

Since we now have an approximate number of Mercury's theta half-wavelengths, fitting its orbital perimeter, we can determine the same paranneter value for each one of the remaining planets, further testing the consistency of the wave model for the Solar System.

In order to do the calculations, we will use a generalized gravitational version of de Broglie's wavelength-linear momentum formula

$$
v=\frac{h_{G}}{\lambda}
$$

This expression does not involve the planetary mass, since only the planetary linear velocity was found to be quantified, according to an exponential expression as in (4).

From (10) and (12) we get for Mercury's theta wavelength

$$
\lambda_{1}=\frac{2}{3} \pi r_{1}
$$

Substituting in (13) the values for Mercury's velocity and wavelength, we get: 


$$
h_{G} \cong 5,758 \times 10^{15} \quad\left(\mathrm{~m}^{2} / \mathrm{s}\right)
$$

We will call this parameter the planetary gravitational constant for the Solar System.

Presumably, this parameter will vary for each gravitational system. Relation (13), on the other hand, can be considered valid for any planetary system, once the value of $h_{G}$ has beem determined for each particular system under interest.

To determine the integer number of theta half-wavelengths for each planet, we combine relation (10) with relation (13) to get

$$
n_{i}=\frac{4 \pi r_{i} v_{i}}{h_{G}}
$$

The following table shows each planet's integer number of half-wavelengths in its perimeter.

Table 3. Planet's integer number of half-wavelengths in its perimeter.

\begin{tabular}{cccccccc}
\hline Mercury & Venus & Earth & Mars & Jupiter & Saturn & Uranus & Neptune \\
\hline 5.99 & 8.26 & 9.73 & $\mathbf{1 1 . 9 9}$ & 22.26 & 30.35 & 42.63 & 52.98 \\
\hline
\end{tabular}

These can be rounded to units, giving the sequence $6,8,10,12,22,30,43,53$.

Interestingly enough this sequence also follows an exponential distribution as shown in Figure 4.

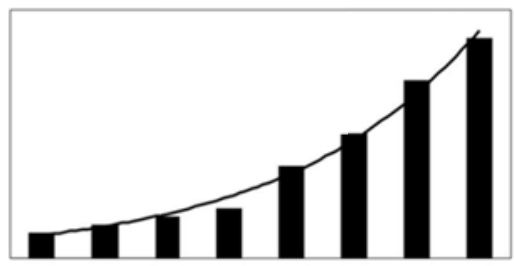

Figure 4. Exponential fitting for the integer numbers of half wavelengths.

As the planet stands more distant from the Sun, more theta half-wavelengths are needed to equal the planet's orbital perimeter.

It should be mentioned that from (16) we may easily derive the expression for the angular momentum of each planet, divided by its mass, which will also be quantified according to relation

$$
L_{i}^{\prime}=n_{i} \frac{h_{G}}{4 \pi}\left(\mathrm{m}^{2} / \mathrm{s}\right)
$$

\section{Conclusion}

Under reasonable assumptions it has been suggested that there is physical evidence for the existence of gravitational planetary pilot waves, manifested through the Titius-Bode Law. A strong argument supporting the previous statement, is that this empirical expression, similar to atonnic empirical laws like Balmer's and others, once expressed as a sequence of integer numbers, may be interpreted as strong evidence for the existence of wave-like phenomenon. As it stands, the planetary orbital periods also follow an exponential distribution, being quantified in the sense previously explained. In future work we intend to extend our model, accounting for this further regularity.

\section{References}

1. Blagg, Mary Adela, "On a suggested substitute for Bode's Law", Mon. Not. R. Astron. Soc. 73, 414-22 (1913).

2. Richardson, D. E., "Distances of planets from the Sun and of satellites from Their primaries in the satellite systems of Jupiter, Saturn, and Uranus", Pop. Astron. 53, 14-26 (1945). 
3. Nieto, Michael Martin. The Titius-Bode Law of Planetary Distances: Its History and Theory. Edited by D. Ter Haar. Pergamon, 1972

4. de Broglie, Louis, "La mécanique ondulatoire et la structure atomique de la matière et du rayonnement" in Le Journal de Physique et le Radium (6), 8, 225-41, (Paris: 1927).

5. Croca, J. R., Eurhythmic Physics or Hyperphysics. Saarbrücken: LAP Lambert Academic Publishing, 2015.

6. Planetary Fact Sheet - Metric webpage, Available: http://nssdc.gsfc.nasa.gov/planetary/factsheet/ 